\title{
DESIGN OF WELDING SYSTEM FOR LITHIUM BATTERY BASED ON
}

\section{PLC}

\author{
Huang Tao ${ }^{1}$, Wang Minghong ${ }^{2}$ \\ ${ }^{1}$ Post Graduate Student, Department of Mechanical Engineering, Shanghai University of Engineering Science, \\ Shanghai, China \\ ${ }^{2}$ Associate Professor, Department of Mechanical Engineering, Shanghai University of Engineering Science, Shanghai, \\ China
}

\begin{abstract}
Design a set of welding equipment for lithium battery by spot welding techniques. The motion mechanism of the equipment's mechanical system are composed of XY linear modules and a cylinder. The control system of the equipment is composed of PLC, servo driver, servo motor and touch screen. The plain position of the welding electrodes is controlled by the servo system. The height of the welding electrodes is adjusted by the cylinder. In addition, the continuous welding of lithium battery is realized by the teaching function of touch screen. After a period of practical application, the system was stable and reliable and realized fast and efficient welding of lithium battery.
\end{abstract}

Keywords: Welding System; Spot Welding; PLC; Lithium Battery -***

\section{INTRODUCTION}

With the rise of labor costs and the increasing requirements of product quality, the traditional labor-intensive manufacturing industries which depend on manual operation are faced with enormous product costs and pressure of quality. The use of automated production mode is an inevitable trend of the traditional manufacturing industries to break through the bottlenecks of its development and promote their abilities in competitiveness, which has become the consensus of the current manufacturing enterprises, especially small and medium manufacturing enterprises. Automatic spot welding machine is used to replace the traditional manual welding operation which has a positive significance to improve the automation level of the manufacturing enterprises [1-3].

\section{TECHNICALREQUIREMENTS}

AND

STRUCTURE OF THE MECHANICAL SYSTEM FOR SPOT WELDINGMACHINE

\subsection{Technical Requirements for Spot Welding}

\section{Machine}

The main technical requirements for spot welding machine are shown as follows:

(1) The biggest size of the lithium battery pack is $400 \mathrm{~mm} * 300 \mathrm{~mm}$.

(2) Welding parameters can be set by teaching programming. There are 20 sets of welding parameters and single set of the parameters are up to 200 points.
(3) Security protections and real-time state monitoring functions should be equipped.

(4) Position accuracy is $0.02 \mathrm{~mm}$, single point welding speed $<=1$ s.

(5) Manual and automatic control functions should be equipped.

(6) Equipment power supply is AC $220 \mathrm{~V}$.

\subsection{The Structure of Mechanical System}

The structure diagram of the spot welding machine is shown in Fig 1.The electric controlling board 11 is for the installation of electrical components such as PLC, Servo drivers, DC power and etc.as well as pneumatic components such as solenoid valve, manifold valves, filter and etc..The lifting valve 5 is a cylinder with magnet and two magnetic switches used to detect its signal to initial and to be in position .The cylinder 7 is used to clamp the lithium battery package 4 . The copper electrodes 6 ought to be oxidized to result in poor welding in the welding process, so it should be moved to the position of the grinding groove 3 to grind itself by ordinary sand paper. The welding power 1 could be selected as Miyagi MDA-8000B or MDA-8000B transistortype .The photoelectric switch 12, 14 are used to detecting the limitation signals of $\mathrm{X}$ axis and the photoelectric switch 13 are used for the detection of the origin signal of $X$ axis [4].The $Y$ axis also has three photoelectric switches with the same functions as the $\mathrm{X}$ axis do. It is needed to emphasize that the photoelectric switches detecting the origin signals of the $\mathrm{X}$ and $\mathrm{Y}$ axis are connected to the same terminal of the PLC [5-6]. 


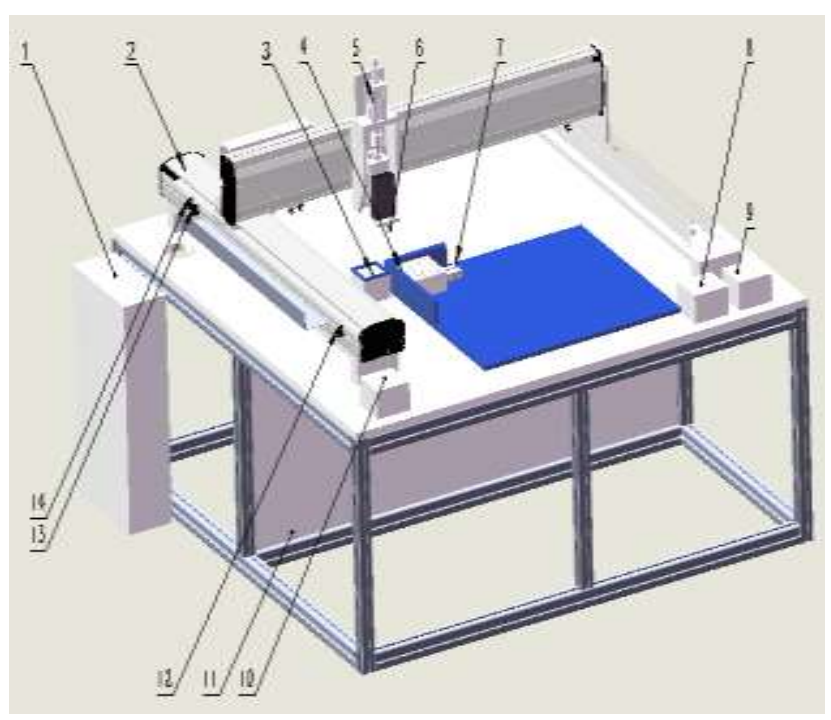

1-WeldingPower, 2-Linear Modules, 3-Grinding Groove, 4Lithium Battery Package, 5-Lifting Valve, 6-Copper

Electrodes, 7-Cylinder, 8-Start Button, 9-Pause / Restart Button, 10-Emergency Button, 11-Electric Controlling

Board, 12、13、14-Photoelectric Switch

Fig-1: Structure Diagram of the Spot Welding Machine

\section{COMPOSITION OF CONTROL SYSTEM AND CONTROL PROCESS OF PLC}

\subsection{Hardware Configuration of Control System}

The servo control system is composed of Delta DVP32EH00M3 PLC, Panasonic MBDHT2510E servo driver,MHMD042G1U servo motor that drives the $\mathrm{X}$ axis,MBDHT1510E servo driver and MHMD022G1U servo motor that drives the $\mathrm{Y}$ axis. The I/O configuration of PLC is shown in Table-1.

\subsection{I/O Distribution of PLC}

Fig-2 shows the I/O distribution of PLC. The number 23 and 24 in the diagram correspond to the GOOD and NG output terminals of Miyagi welding power. The point $\mathrm{Y} 0$ and $\mathrm{Y} 1$ of the PLC control the pulse and direction of the $\mathrm{X}$ axis. Respectively, the point Y2 and Y3 control the pulse and direction of the $\mathrm{Y}$ axis. The pulse output terminals of phase $\mathrm{A}$ and $\mathrm{B}$ in $\mathrm{X}$ axis servo driver are connected with the point X0 and X1 which correspond to the high-speed counter C251 of the PLC. The pulse output terminals of phase A and $\mathrm{B}$ in $\mathrm{Y}$ axis servo driver are connected with the point of $\mathrm{X} 4$ and $X 5$ which correspond to the high-speed counter C252 of PLC[7]. The four limitation signals of $\mathrm{X}$ and $\mathrm{Y}$ axis are connected with the point X17 in parallel. The origin signals of $\mathrm{X}$ and $\mathrm{Y}$ axis need to be connected with the high-speed input port X10 and X11.In addition, the pause/reset and emergency button are connected with the high-speed input port X14 and X15 because hardware connected with highspeed input port can improve the response rate of the system[8].

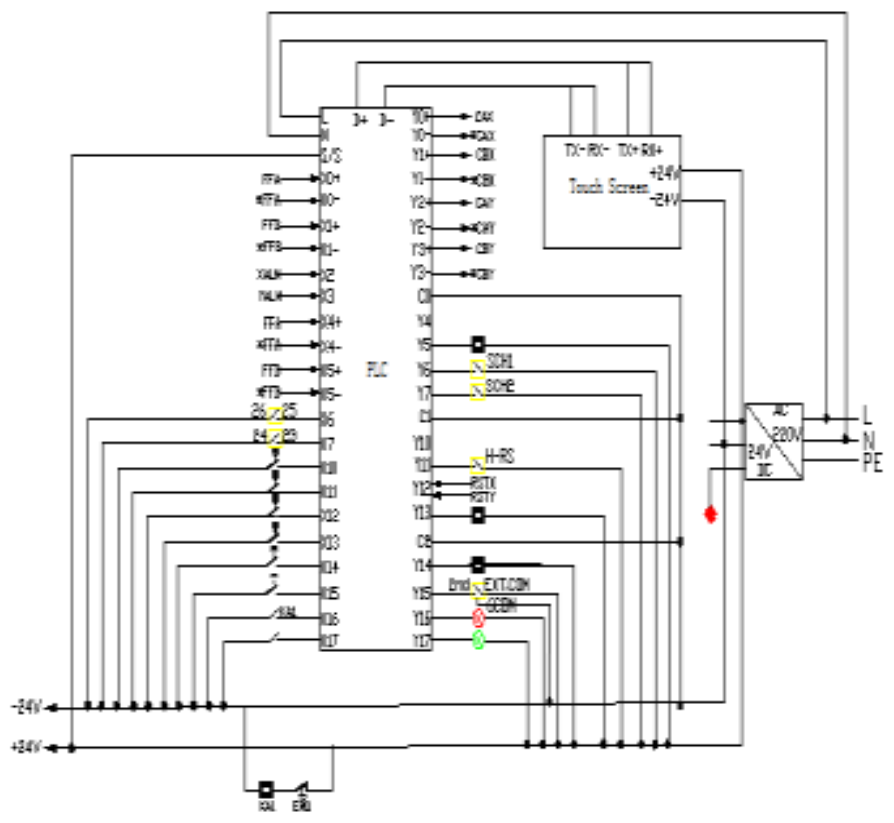

Fig-2: I/ O Distribution Diagram of PLC

\subsection{Control Process of PLC}

Owing to the use of incremental encoders in the control system, it is needed to press the reset button on the touch screen before starting the welding system. After pressing the reset button, the point Y12 will obtain electricity, making the servo drivers of XY axis reset. Subsequently the XY axis will go back to their origins after which press the start button while the point Y5 will obtain electricity, making the cylinder which is controlled by solenoid valve clamp the lithium battery pack and the copper electrodes will move to the position over the lithium battery. When the copper electrodes is over the lithium battery, the point Y14 will obtain electricity making the lifting valve move to the position which is set by magnetic switch. Then the point X12 will obtain electricity after which the point Y15 will obtain electricity, making the copper electrodes begin welding the lithium battery. During the process of welding, the Y13 point is getting electricity, making the windpipe which is controlled by solenoid valve cool the lithium battery being welded. If the point X12 does not obtain electricity at the set time, the point Y16 would obtain electricity, making the red light flash and the buzzer of the touch screen alarm. After finishing welding a single lithium battery, the point $\mathrm{X} 7$ will obtain electricity after which the point Y14 will lose electricity, making the lifting valve go back to its initial position. Then the point X16 will obtain electricity and the system will begin welding the next lithium battery. If the point $\mathrm{X} 7$ and $\mathrm{X} 16$ do not obtain electricity at the set time, the point Y16 will also obtain electricity, making the red light flash and the buzzer of the touch screen alarm. During the process of welding, every alarm could be recorded on the touch screen. If the red light flashes, the system will stop working and the point Y11 will obtain electricity, making the welding power reset. During the process of handling the trouble, We can press the alarmclear button of the touch screen to stop the red light flash. If the red light continues flashing after releasing the alarmclear button, it shows that the trouble have not been handled. 
Table-1:I/O Distribution Table

\begin{tabular}{|l|l|l|l|}
\hline input & & Output & \\
\hline X0 & Phase A of X Axis Encoder & Y0 & Phase A Pulse of X Axis \\
\hline X1 & Phase B of X Axis Encoder & Y1 & Phase B Pulse of X Axis \\
\hline X2 & Servo Alarm of X Axis & Y2 & Phase A pulse of Y Axis \\
\hline X3 & Servo Alarm of Y Axis & Y3 & Phase B pulse of Y Axis \\
\hline X4 & Phase B of Y Axis Encoder & Y4 & \\
\hline X5 & Phase A of Y axis Encoder & Y5 & Clamping Cylinder \\
\hline X6 & Welding Power Alarm & Y6 & \\
\hline X7 & Welding OK & Y7 & \\
\hline X10 & Origin of Xaxis & Y10 & \\
\hline X11 & Origin of Y axis & Y11 & Welding Power Reset \\
\hline X12 & Welding Cylinder In Position & Y12 & X/Y Servo Reset \\
\hline X13 & Start Signal & Y13 & Air Cooling \\
\hline X14 & Pause / Restart Button & Y14 & Lifting Valve \\
\hline X15 & Emergency Button & Y15 & Welding Trigger Signal \\
\hline X16 & Welding Cylinder Initial Position & Y16 & Red Light \\
\hline X17 & Limitation Signals of X and Y axis & Y17 & Green Light \\
\hline
\end{tabular}

\section{ALGORITHMS AND PROGRAM OF CONTROL SYSTEM}

Delta WPL Soft software is the tool for programming. The main program of the system monitors the various parameters in the welding process. The subroutines mainly realize the functions of system reset, matrix-teaching, welding, electrodes grinding and alarm processing. We mainly discuss the part of initialization program in the main program, electrodes' grinding program and matrix-teaching Algorithms as follows.

\subsection{Initialization Program}

In Delta EH2/SV models, counter C251 is corresponded to the high-speed counter HHSC0 while counter C252 is corresponded to the high-speed counter HHSC1.Each highspeed counter has three kinds of counting modes, namely, one phase one input(pulse/direction mode), one phase two input(forward/ reverse mode), two phase two input(A/B phases mode).According to the connections between the servo drivers and the high-speed input terminals, It is known that HHSCO and HHSC1 adopt the third mode. Each HHSC is provided with a reset and start signal from external input. For example, the reset and start input of HHSC0 is the point $\mathrm{X} 2$ and X3.Similarly,the reset and start input of HHSC1is the point X6and X7. When using the high-speed counter, if the external reset and start control signal input are not used, the register M1264/M1265 and M1266/M1267 can be used to close the actions of them. So the point X2/X3 and X6/X7 can be used as ordinary input terminals.

In Delta EH2/SV/EH3/SV2 models, the special registers D1221 and D1220 are used for the phase setting of highspeed input terminals of $\mathrm{CH} 0$ (Y0, Y1) and $\mathrm{CH} 1$ (Y2, Y3).There are four kinds of setting modes. Take $\mathrm{CH} 0(\mathrm{Y} 0, \mathrm{Y} 1)$ as an example, they are output of the point
Y0, output of phase $\mathrm{A}$ and $\mathrm{B}$ (A is prior to $\mathrm{B}$ ), output of phase $A$ and $B(B$ is prior to $A)$ and output $Y 1$. The corresponding selection method of the four modes is to set number of the special register D1220 as zero to three. Because the servo motors of $\mathrm{X}$ and $\mathrm{Y}$ axis adopt the pulse/direction control mode, it is needed to set D1220 and D1221 as zero[9].In summary, the initialization program is shown in Fig-3.

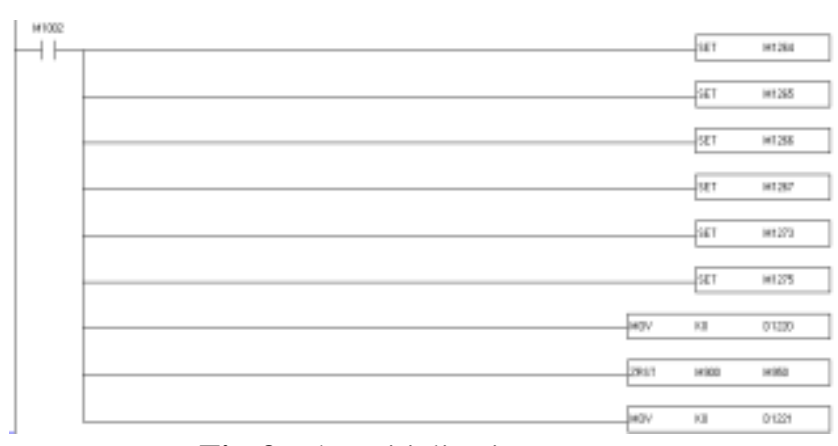

Fig-3: The Initialization Program

\subsection{Algorithms for Circle Interpolation}

In the welding process, the welding electrode ought tooxidize and result in the problem of unhealthy welding. Therefore, it is needed to grind the copper electrodes at a certain time. The arc interpolation function is realized by the instruction DCIMR. As is shown in Fig-4, point A is the origin. From the point $\mathrm{A}$ to $\mathrm{B}$, the pulse number in $\mathrm{X}$ and $\mathrm{Y}$ direction is $a, a$.From the point $\mathrm{B}$ to $\mathrm{C}$, the pulse number in $\mathrm{X}$ and $\mathrm{Y}$ direction is $a,-a$. From the point $\mathrm{C}$ to $\mathrm{D}$, the pulse number in $\mathrm{X}$ and $\mathrm{Y}$ direction is $-a,-a$. And from point $\mathrm{D}$ to $\mathrm{A}$, the pulse number in $\mathrm{X}$ and $\mathrm{Y}$ direction axis is $-a,-a$.Take the arc from $\mathrm{A}$ to $\mathrm{B}$ as an example, if the number of $a$ is stored in the register D0,the corresponding instruction is DCIMR D0 D2 N D in which D and N refer to the resolution and pulse output device. 


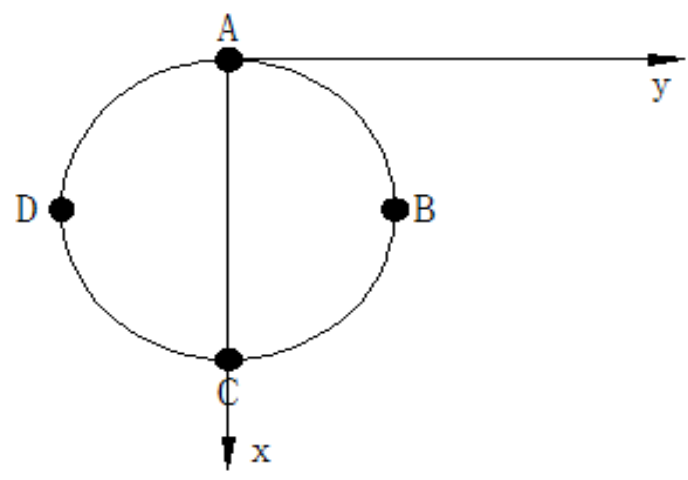

Fig-4: Diagram of Circle Interpolation

\subsection{Algorithms of Matrix-teaching}

The number of rows and columns of lithium battery package can be input in the matrix-teaching interface. The corresponding register addresses can be set as the register D0 and D2. As depicted in Fig-5, the x/y position of point A can be stored in the register D4 and D6. The $\mathrm{x} / \mathrm{y}$ position of point $\mathrm{B}$ can be stored in the register D8 and D10.The $\mathrm{x} / \mathrm{y}$ position of point $\mathrm{C}$ can be stored in the register D12 and $\mathrm{D} 14[10]$. So it can be concluded that $l_{x}=\frac{(D 12-D 4)}{D 0-1}$, $l_{y}=\frac{(D 10-D 6)}{D 2-1}$

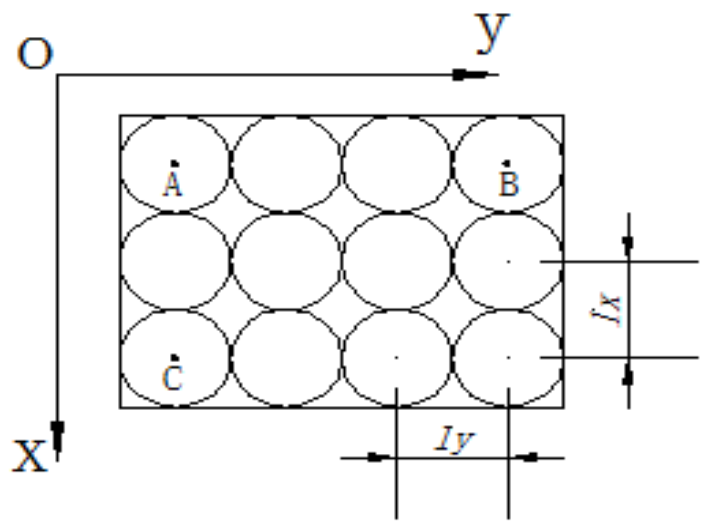

Fig-5: Diagram of Matrix-teaching

\section{COMPOSITION AND OPERATION METHOD}

\section{OF HMI}

\subsection{Composition of HMI}

Delta DOP Soft software is used to edit HMI interface which is mainly composed of the main-control, matrixteaching, parameter-setting and alarm-recording interfaces.Fig-6 shows the main-control, matrix-teaching and parameter-setting interfaces.

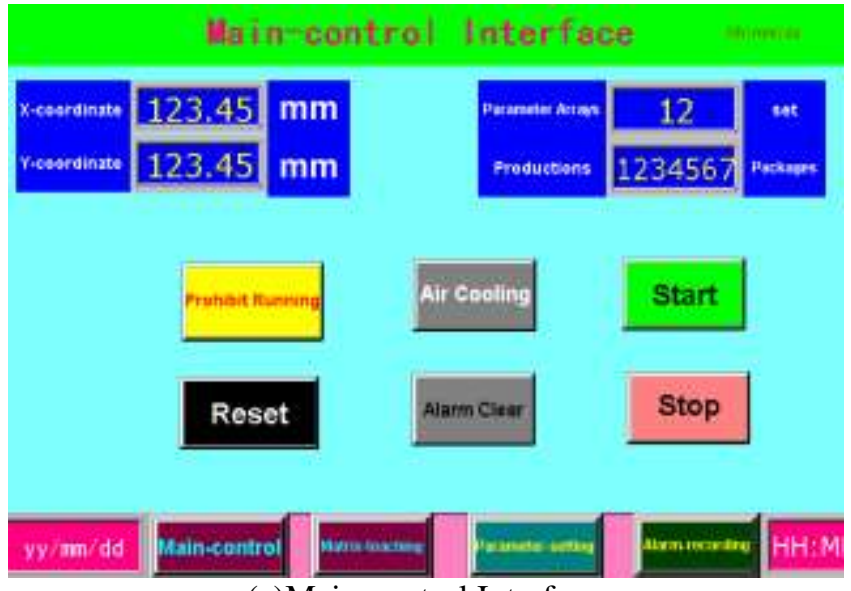

(a)Main-control Interface

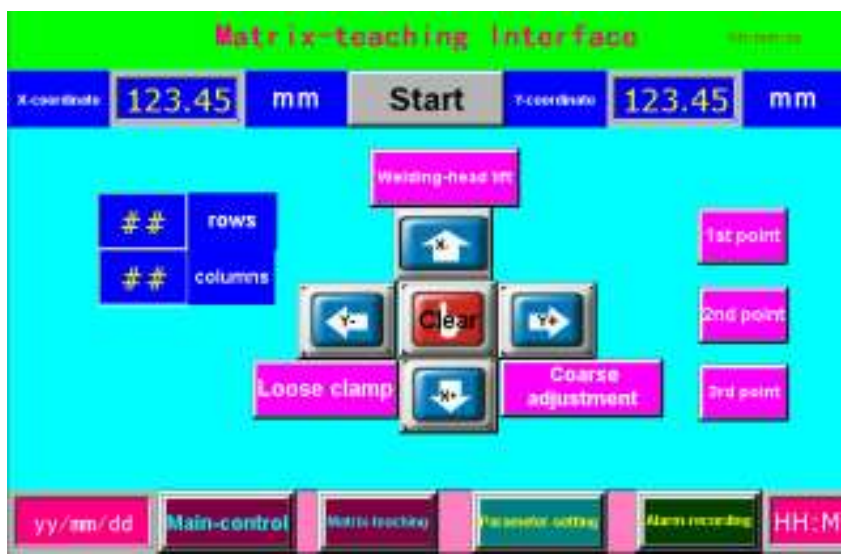

(b)Matrix-teachingInterface

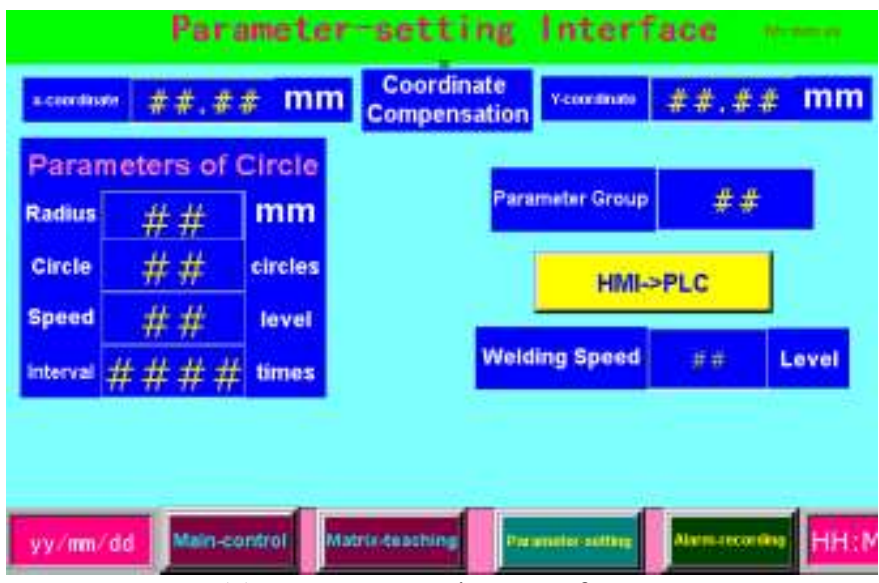

(c)Parameter-setting Interface

Fig-6: Composition of HMI

\subsection{Basic Operation Method of HMI}

Firstly, press the reset button in the main-control interface to reset the system. Secondly, choose to enter the matrixteaching interface to finish the work mentioned above and download them to PLC. Then set the welding parameters in the parameter-setting interface and download them to PLC. Finally press the reset button in the main-control interface to reset the system again, then press the start button after the $\mathrm{XY}$ axis in its initial position to make the system run . 


\section{CONCLUSION}

In this paper, a kind of general automatic spot welding system was designed according to the actual requirements in lithium battery industry. The use of the formula function of touch screen achieves the continuous and fast welding of the lithium battery. After a period of production, the automatic spot welding system is stable and reliable, fully met the expected performance requirements. To a great extent, it has improved the production efficiency, reduced the production costs of enterprises and achieved good economic benefits.

\section{REFERENCES}

[1]. Shiyu LI,Aiqing CHEN. Research and Development of Lithium Battery Automatic Spot Welding Equipment[J]. Journal of Electric Tool ,2014,(6):9-13.

[2]. MengLong ZHOU. Development and prospect of welding equipments home and abroad[J].Journal of WeldingTechnology,2005,34(6):1-8.

[3]. Gewang SHUAI,Pingjian ZHOU,Jianbin LIU.Research Progress and Trends in Resistance Spot Welding Electrode[J].

Materials Review,2015,29(7):59-62.

[4]. Minghui QIANG,XiaoHE.A Servo Control System Based on PLC Positioning Module EM253[J].Journal of Electrical Automation,2014,(3):3-4.

[5]. Zhengzhong LI,Guangping ZHOU,Zhaofeng LIANG,Xinhong CHEN.Design of ultrasonic metal-welding machine for lithium battery[J].Journal of Welding Technology,2012,41(1):40-42,55.

[6]. JuQIU,Mingrui WANG,Jing ZHAI,Peisheng LI, Yuanyuan FU.Application of five axis linkage laser welding system in automotive lithium battery welding[J].Journal of Manufacturing Automation, 2016,38(6):84-85.

[7]. Shuguang HOU,Hui HUANG,Yang YANG,Huafeng LIU.The design and development of specific spot-welding bench[J].IndustrialInstrumentation\&Automation,2012,(2):69 -73 .

[8]. Feng PAN,Dajun CHEN.The PLC Control of Spot Welding Machine[J].MECHANICAL \& ELECTRICAL ENGINEERING MAGAZINE,2001,18(6):26-29.

[9]. Jie XIU,Yulan LI,Shixu XU.Design of PLC Control System for Feeder[J].Journal of Industrial Control Computer,2012,25(4):100-101.

[10]. Dong SHI,Yong ZHANG,Tianju CHENG.Design of the Welding Control System Based on PLC and Touch Screen[J].Journal of Electrical Automation,2012,34(6):5152,68 .

\section{BIOGRAPHIES}

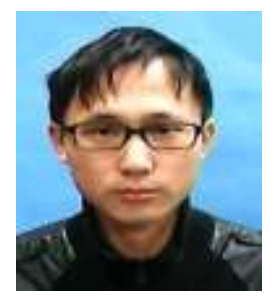

Tao Huang. The author is a graduate student at Shanghai University of Engineering Science and mainly Engages in the research of automation . Email:635054169@qq.com

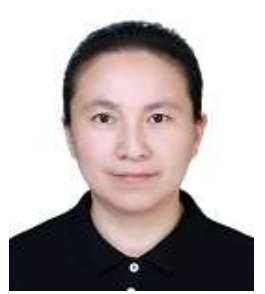

Minghong Wang: As an associate professor, her research interests are issues of professional fields and training of practical capability for university students. She is now specializing in the development of mechanical design, Manufacturing and automation. 